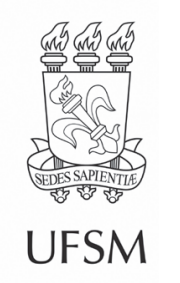

\title{
Artigos
}

\section{Aplicação do modelo MGB-IPH na bacia hidrográfica do Rio Pardo em diferentes cenários de uso e ocupação do solo}

\author{
Application of the MGB-IPH model in the Pardo River hydrographic basin \\ in different scenarios of use and occupation of the soil

\section{Rodrigo Lacerda Brito Neto ${ }^{\perp}$, Cristiano Tagliaferre ${ }^{\Perp} \odot$, Odair Lacerda Lemos "๑, Felizardo Adenilson Rocha "II $₫$,Alessandró de Paula "๑} \\ ' Universidade Federal Rural de Pernambuco, Recife, PE, Brasil \\ " Universidade Estadual do Sudoeste da Bahia, Vitória da Conquista, BA, Brasil \\ III Instituto Federal de Educação, Ciência e Tecnologia da Bahia, Vitória da Conquista, BA, Brasil
}

\section{RESUMO}

As alterações causadas pela ação do homem ao meio ambiente podem influenciar a disponibilidade de água em bacias hidrográficas, e mensurar os impactos causados por essa ação é imprescindível para a tomada de decisões por órgãos gestores. Dessa forma, o objetivo deste estudo foi realizar a calibração e validação do modelo MGB-IPH para simular o comportamento da vazão na bacia hidrográfica do rio Pardo em diferentes cenários de uso e ocupação do solo. Para isso, foram usadas informações de uso e ocupação do solo que revelaram que a bacia hidrográfica em estudo teve uma redução em sua área de floresta e aumento das áreas dedicadas às atividades agropecuárias, entre os anos de 2001 e 2016. Com essas informações acerca da bacia e com os dados climáticos e os tipos de solos disponíveis, foram realizadas a calibração e a validação do modelo, que teve seus desempenhos verificados. As mudanças nas vazões mensais médias, totais, máximas e mínimas simuladas pelos diferentes cenários foram comparadas por meio de teste de média para amostras dependentes e avaliação das vazões anuais máximas, médias e mínimas dos valores testados. O MGB-IPH foi validado de forma satisfatória para a estação 53880000 da Agência Nacional de Águas, tendo problemas de subestimativa de picos de vazões máximas, porém conseguindo representar adequadamente os dados observados em termos da sazonalidade das vazões, da diferenciação de anos mais úmidos e anos mais secos, e dos picos de cheias de média e pequena intensidade. O modelo foi sensível ao identificar o impacto das mudanças do uso e ocupação do solo nas vazões simuladas para a bacia hidrográfica do rio Pardo, com o cenário de 2016 apresentando maiores valores de vazões mensais médias, totais, máximas e mínimas que as encontradas no cenário de 2001.

Palavras-chave: Modelo hidrológico; Vazão; MapBiomas 


\section{ABSTRACT}

The changes caused to the environment by human actions can influence the availability of water in hydrographic basins, and measuring the impacts caused by these actions is essential for decisionmaking by management bodies. Thus, the aim of this study was to perform the calibration and validation of the MGB-IPH model to simulate the flow behavior of the Pardo River hydrographic basin in different scenarios of use and occupation of the soil. For this purpose, we used information on the use and occupation of the soil, revealing a reduction in the forest area of the hydrographic basin under study and an increase in the areas dedicated to agricultural activities, between 2001 and 2016. With this information about the basin, with the climatic data and the types of soils available, the model was calibrated and validated, and its performance was verified. The changes in the average, total, maximum, and minimum monthly flow rates simulated by the different scenarios were compared by average test for dependent samples, and we performed the evaluation of the maximum, average, and minimum annual flow rates of the values tested. The MGB-IPH has been satisfactorily validated for the Agência Nacional de Águas, station 53880000, presenting problems with the underestimation of maximum peak flow rates, but managing to adequately represent the observed data in terms of flow rate seasonality, differentiation between wetter and drier years, and between medium and small intensity peak floods. The model was sensitive in identifying the impact of changes in the use and occupation of the soil in simulated flow rates for the Pardo river basin, with the 2016 scenario showing higher values of average, total, maximum, and minimum monthly flow rates compared to those found in the 2001 scenario.

Keywords: Hydrological model; Flow rate; MapBiomas

\section{INTRODUÇÃO}

O processo de antropização de uma região envolve primordialmente o acesso à água, pois é por meio desta que se desenvolverão as práticas rurais e urbanas nas quais o ser humano, por intermédio da sua força de trabalho, ocupa e modifica o ambiente (MELO, 2016). Nesse processo de ocupação, são definidos os usos do solo; áreas antes pertencentes às vegetações naturais poderão ser destinadas tanto às práticas agrícolas, urbanização, construção de estradas, como para a conservação e preservação dos recursos naturais.

Se realizada de forma desordenada, essa ocupação pode gerar problemas geoambientais que colocam em risco a comunidade local e regional, sendo incompatível com as características geofísicas do ambiente (MELO, 2016). A região tratada neste estudo é a bacia hidrográfica, e nesta, as consequências da diminuição de áreas de florestas e vegetações naturais não florestais são o aumento do escoamento 
superficial, perda de solo por erosão, bem como a redução da evapotranspiração e da interceptação das águas pluviais, promovida pela vegetação nativa (SILVA; SILVA; SOUZA, 2016; LOTZ; OPP; HE, 2017). A expansão de áreas destinadas a atividades agropecuárias tem sido relacionada à redução nas taxas de infiltração e aumento da densidade aparente, provenientes de práticas não conservacionistas, como a utilização de máquinas pesadas e pastejo não rotacionado; efeitos que contribuem para a potencialização do escoamento superficial e dificultam o abastecimento dos lençóis freáticos (FEITOSA et al., 2015; SILVA; BIANCHINI; CUNHA, 2016).

Realizar a previsão do comportamento e dos impactos sofridos sobre bacias hidrográficas é um dos desafios dos estudos hidrológicos. Para auxiliar nessa tarefa, Bayer e Collischonn (2013) evidenciam a importância da simulação hidrológica que, segundo os autores, por meio de modelos, possibilita a tomada de medidas preventivas por meio da análise do comportamento de diferentes cenários ou condições de ocupação de solo sobre a bacia, contribuindo assim para a redução dos impactos.

Modelos que permitem a simulação dos processos hidrológicos no tempo e no espaço são chamados de modelos distribuídos. Nesses modelos, Haghnegahdar et al. (2015) informam que os processos hidrológicos são representados em subunidades da bacia hidrográfica, que podem ser células regulares ou irregulares baseadas na topografia, considerando as diferentes classes de respostas hidrológicas (CRHs). Essas CRHs são regiões da bacia que possuem comportamento hidrológico semelhante, que podem incorporar informações de características físicas importantes das bacias, como o relevo e os tipos de solos, bem como sua distribuição no espaço e tempo.

O modelo hidrológico de grandes bacias do Instituto de Pesquisas Hidráulicas da Universidade Federal do Rio Grande do Sul (MGB-IPH) é um modelo distribuído que utiliza uma base física que se divide em dois módulos: um que simula os processos hidrológicos verticais (balanço hídrico no solo, interceptação e evapotranspiração) e outro que simula os horizontais (escoamento superficial, subsuperficial, subterrâneo, propagação da vazão nos rios e na planície de inundação e propagação em reservatórios) 
(BAYER; COLLISCHONN, 2013). A evapotranspiração no modelo é calculada pela equação de Penman-Monteith, e a propagação na rede de drenagem é feita através do método de Muskingum-Cunge.

Entre os softwares de modelagem hidrológica disponíveis, pode-se citar o Sistema de Modelagem Hidrológica (HEC-HMS), desenvolvido pelo Corpo de Engenheiros do Exército Americano; e o SWAT, um modelo hidrológico de domínio público, desenvolvido pelo Agricultural Research Service (ARS), no Grassland, Soil and Water Research Laboratory, em Tempe, Texas, EUA. Ambos são modelos distribuídos, assim como o MGB-IPH, porém o último diferencia-se por ser um modelo criado no Brasil e permitir a recuperação automática de dados de solo e vegetação, por intermédio de imagens de satélite (COSTA, 2014).

O MGB-IPH já foi aplicado em várias regiões do Brasil, dentre elas: na previsão de vazões e simulação hidrológica na bacia hidrográfica do rio dos Sinos-RS (PEREIRA; KAYSER; COLLISCHONN, 2012); para simulações de qualidade da água nas bacias do rio Ijuí-RS, São Francisco-MG e do rio Piracicaba-MG (DE MELO, 2014; FAN, 2013). Também já foi aplicado com sucesso em estudos sobre impactos de mudanças climáticas em bacias hidrográficas, incluindo o rio Grande (NÓBREGA et al., 2011), rio Quaraí, entre o Rio Grande do Sul e o Uruguai (PAIVA; COLLISCHONN; SCHETTINI, 2011) e no rio Paraguai, na região do Pantanal (BRAVO et al., 2014).

Devido à sua vasta aplicação e pela bacia não apresentar informações a respeito de modelagem, este estudo teve como objetivo realizar a calibração e a validação do modelo MGB-IPH para simular o comportamento da vazão na bacia hidrográfica do rio Pardo em diferentes cenários de uso e ocupação do solo.

\section{MATERIAL E MÉTODO}

\section{1 Área de estudo}

A bacia hidrográfica do rio Pardo possui área de drenagem de $32.649 \mathrm{~km}^{2}$. Seu 
rio principal, o rio Pardo, possui sua nascente no município de Montezuma-MG e foz em Canavieiras-BA. É uma bacia longa e grande, que possui uma rede de drenagem com canais de até sexta ordem, baixa densidade de drenagem e um rio principal muito sinuoso. A maior parte de sua área está entre 600 m e 1000 m, com uma grade amplitude altimétrica (BRITO NETO, 2018).

A precipitação média anual varia de 703,72 mm, na região central da bacia, a $1.325,05 \mathrm{~mm}$, mais próximo à foz, sendo que o período mais chuvoso vai de outubro a abril, com precipitação superior a 60 mm (abril) e valores acima de 163 mm (dezembro); e o mais seco vai de maio a setembro, período em que os valores de precipitação média mensais são inferiores a 60 mm (SANTOS, 2017).

\subsection{Criação das classes de resposta hidrológica}

Realizou-se o download, por meio do projeto Topodata (DE MORISSON; DE FÁTIMA, 2012), de oito cenas do Shuttle Radar Topography Mission (SRTM), com resolução espacial de aproximadamente 30 metros, que foram unidas para formar um único modelo digital de elevação (MDE). Esse modelo foi utilizado para a obtenção da rede de drenagem, direções de escoamento, área de captação com exutório no ponto de latitude $-15^{\circ} 38^{\prime} 35^{\prime \prime}$ e longitude -3900'47" e minibacias (área contribuinte de cada trecho de drenagem na qual a água precipitada é conduzida diretamente para aquele segmento) (Figura 2).

As informações do tipo de solo foram retiradas do mapa de solos do Brasil (SANTOS et al., 2011), na escala de 1:5.000.000, obtido online pelo repositório de Informação Tecnológica da Embrapa (Infoteca-e). Trata-se de um mapa em formato PDF, cuja área correspondente à bacia hidrográfica anteriormente delimitada foi recortada e georreferenciada com o auxílio da aplicação ArcMap 10.2. Os tipos de solos foram reclassificados para solos rasos e profundos, da seguinte forma: argissolos, chernossolos, espodossolos e latossolos, como classe de "solo profundo"; e cambissolos, gleissolos, luvissolos e neossolos, como classe de "solo raso". 
Para gerar os mapas de uso e ocupação do solo que serão utilizados como cenários diferentes dentro do modelo, foram usados os dados da coleção 2.3 da plataforma online do Projeto de Mapeamento Anual da Cobertura e Uso do Solo do Brasil (MapBiomas). Realizou-se o downloaddos anos de 2001 e 2016, dos mapas de uso e ocupação para os biomas inseridos na área de captação (Caatinga, Mata Atlântica e Cerrado), na escala de 1:1.000.000, no formato GeoTiff. Para cada ano, os mapas dos três biomas foram utilizados com entradas no programa ArcMap 10.2, por meio do qual foram unidos, reprojetados para UTM e datum SIRGAS2000 e recortados para o formato da bacia. As classes ficaram definidas como: floresta, vegetação natural não florestal, agropecuária, áreas sem vegetação e água. Detalhes sobre a metodologia e classes dentro do MapBiomas podem ser consultados em MAPBIOMAS (2017). Todos os dados foram reprojetados para a escala de 1:5.000.000, no formato GeoTiff, pois essa é a menor escala dos dados obtidos, evitando interferência de se trabalhar com diferentes escalas.

Os mapas dos tipos e usos/ocupação do solo foram então sobrepostos para formar os mapas das classes de respostas hidrológicas (CRHs). Como existem dois mapas de uso e ocupação do solo, foram gerados os mapas de CRHs para os anos de 2001 e 2016, configurando assim os dois diferentes cenários que podem ser visualizados na Tabela 1.

Tabela 1 - Porcentagem das áreas ocupadas pelas classes de respostas hidrológicas para os anos de 2001 e 2016, na bacia hidrográfica do rio Pardo, Brasil

\begin{tabular}{lcc}
\hline \multicolumn{1}{c}{ CRHs } & Área em 2001 (\%) & Área em 2016 (\%) \\
\hline Floresta + solo profundo & 27,58 & 22,78 \\
Floresta + solo raso & 7,70 & 7,46 \\
Vegetação natural não florestal + solo profundo & 0,77 & 1,13 \\
Vegetação natural não florestal + solo raso & 1,04 & 1,20 \\
Agropecuária + solo profundo & 57,40 & 61,36 \\
Agropecuária + solo raso & 4,61 & 4,69 \\
Sem vegetação + solo profundo & 0,74 & 1,20 \\
Sem vegetação + solo raso & 0,04 & 0,04 \\
Água & 0,12 & 0,15 \\
\hline
\end{tabular}

Fonte: Autores (2020) 


\subsection{Criação dos projetos de calibração e validação}

Por meio do mapa das minibacias foi gerado um arquivo shapefile, no formato de pontos que se localizam no centro geométrico (centroide) de cada minibacia (Figura 1).

Figura 1 - Mapa das minibacias, das sub-bacias, das estações pluviométricas e fluviométricas na bacia hidrográfica do rio Pardo, Brasil

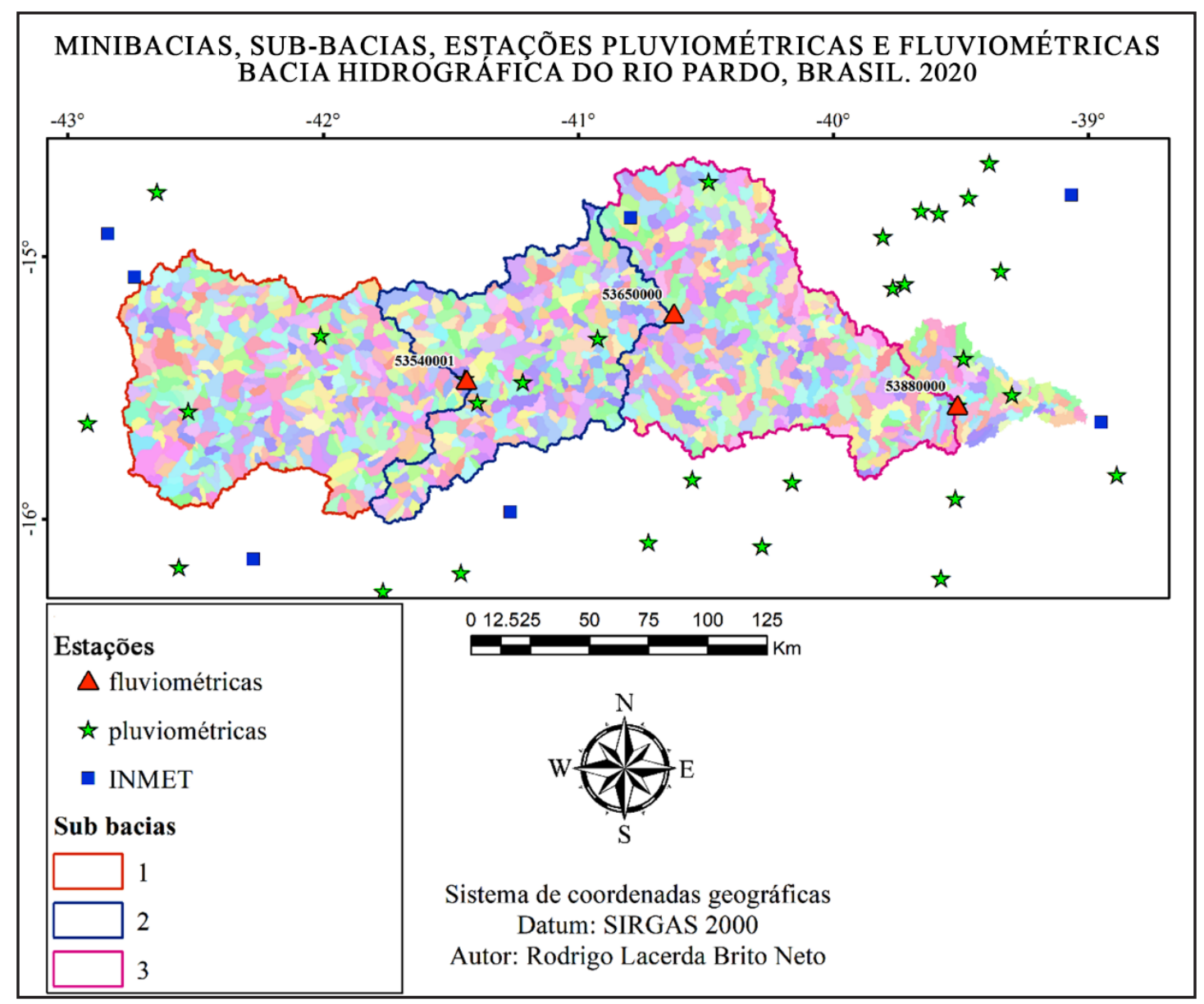

Fonte: Autores (2020)

Os dados diários de chuva foram obtidos do banco de dados da HidroWeb da Agência Nacional de Águas (ANA), por meio do plug-in ANA data acquisition, presente no pacote de ferramentas do MGB-IPH. Realizou-se o download para 30 estações (Figura 2) e, observando-se a disponibilidade de dados, selecionou-se o período de 01/01/2000 a 31/12/2010. 
Por meio do portal HidroWeb da ANA também foram obtidos dados diários de vazões observadas de 01/01/2000 a 31/12/2010, para três postos fluviométricos: 53540001, 53650000 e 53880000.A escolha desses postos foi devido à disponibilidade de dados eessas estações foram utilizadas para delimitar as sub-bacias com exutórios concomitantes às suas localizações (Figura 2), sendo que, no sentido da jusante, têmse: asub-bacia 1, exutório na estação 53540001; a sub-bacia 2, exutório na estação 53650000; e a sub-bacia 3, exutório na estação 53880000. As vazões observadas nos postos fluviométricos foram utilizadas dentro do MGB-IPH para compararàs vazões simuladas e verificar a qualidade da simulação em cada sub-bacia.

Os dados de umidade relativa, insolação, pressão atmosférica, temperatura e velocidade do vento foram obtidos por meio das normais climatológicas (1981-2010) do Instituto Nacional de Meteorologia do Brasil (INMET) e inseridos na base de dados do MGB-IPH para as sete estações indicadas na Figura 2. Esses dados foram transformados em dados diários e então interpolados para cada centroide da minibacia.

Na calibração do modelo MGB-IPH foram considerados parâmetros de vegetação e parâmetros de solo. Os parâmetros de vegetação estão associados à vegetação existente em cada $\mathrm{CRH}$, que são o albedo, a altura média da vegetação, o índice de área foliar (IAF) e a resistência superficial em boas condições de umidade do solo. No modelo, todos esses parâmetros, juntamente com o arquivo de dados de clima interpolados,foram utilizados no cálculo da evapotranspiração pelo método de Penman-Monteith.

Os parâmetros de vegetação utilizados para a simulação da bacia hidrográfica do rio Pardo encontram-se na Tabela 2. Os valores foram escolhidos de acordo com Bayer e Collischonn (2013), que fazem uma vasta revisão dos parâmetros do modelo MGB-IPH.

Os parâmetros de solo também estão associados às CRHs e são alteráveis durante o processo de modelagem, sendo essas alterações que caracterizam o processo de calibração. São parâmetros de solo: armazenamento máximo de água 
na camada de solo; relação entre saturação e armazenamento do modelo utilizado para balanço de água no solo; escoamentos subsuperficial e subterrâneo; tamanho dos poros do solo; fluxo ascendente do aquífero; balanço de água no solo, quando considera-se que a água subterrânea pode retornar por fluxo ascendente para a camada superficial; e coeficientes de ajuste para o tempo de retardo dos reservatórios lineares nas minibacias.

Tabela 2 - Parâmetros de vegetação utilizados no MGB-IPH para a bacia do rio Pardo, Brasil

\begin{tabular}{|c|c|c|c|c|}
\hline CRHs & Albedo & IAF & $\begin{array}{l}\text { Altura média da } \\
\text { vegetação }(\mathrm{m})\end{array}$ & $\begin{array}{c}\text { Resistência } \\
\text { superficial (s/m) }\end{array}$ \\
\hline Floresta + solo profundo & $0,10-0,18$ & $1,50-4,00$ & 12,00 & $70-180$ \\
\hline Floresta + solo raso & $0,10-0,18$ & $1,50-4,00$ & 12,00 & $70-180$ \\
\hline $\begin{array}{l}\text { Vegetação natural não } \\
\text { florestal + solo profundo }\end{array}$ & $0,18-0,23$ & $0,50-1,00$ & 1,00 & $60-80$ \\
\hline $\begin{array}{l}\text { Vegetação natural não } \\
\text { florestal + solo raso }\end{array}$ & $0,18-0,23$ & $0,50-1,00$ & 1,00 & $60-80$ \\
\hline $\begin{array}{l}\text { Agropecuária + solo } \\
\text { profundo }\end{array}$ & $0,20-0,26$ & $0,50-4,00$ & 0,50 & $40-80$ \\
\hline Agropecuária + solo raso & $0,20-0,26$ & $0,50-4,00$ & 0,50 & $40-80$ \\
\hline $\begin{array}{l}\text { Sem vegetação + solo } \\
\text { profundo }\end{array}$ & $0,10-0,35$ & 1,00 & 0,10 & 100,00 \\
\hline $\begin{array}{l}\text { Sem vegetação + solo } \\
\text { raso }\end{array}$ & $0,10-0,35$ & 1,00 & 0,10 & 100,00 \\
\hline Água & 0,08 & 1,00 & 0,00 & 0,00 \\
\hline
\end{tabular}

Fonte: Autores (2020)

A calibração foi feita inicialmente por meio da modificação manual dos parâmetros de solo para cada uma das sub-bacias até a obtenção de um bom ajuste entre as vazões observadas e simuladas. Posteriormente, essa mesma configuração de parâmetros de solo foi utilizada como entrada na calibração automática presente no MGB-IPH. O período selecionado foi de janeiro de 2000 a dezembro de 2005.

A validação do modelo foi feita após o processo de calibração e consistiu na manutenção dos mesmos parâmetros da calibração para o período de janeiro de 2006 a dezembro de 2010. 


\subsection{Análise de desempenho e estatística}

Os resultados da calibração e validação tiveram seus desempenhos verificados por meio do coeficiente de eficiência de Nash Sutcliffe (ENS) e do coeficiente de Nash Sutcliffe dos logaritmos das vazões (ENSlog), conforme podem ser observados nas Equações (1) e (2), respectivamente. Verificou-se ainda o erro percentual de volumes (E (\%)), pela Equação (3).

$$
\begin{aligned}
& \mathrm{ENS}=1-\frac{\sum_{\mathrm{t}=1}^{\mathrm{nt}}\left(\mathrm{Q}_{\mathrm{obs}}(\mathrm{t})-\mathrm{Q}_{\mathrm{cal}}(\mathrm{t})\right)^{2}}{\sum_{\mathrm{t}=1}^{\mathrm{nt}}\left(\mathrm{Q}_{\mathrm{obs}}(\mathrm{t})-\overline{\mathrm{Q}_{\mathrm{obs}}}\right)^{2}} \\
& \mathrm{ENS}_{\mathrm{log}}=1-\frac{\sum_{\mathrm{t}=1}^{\mathrm{nt}}\left(\log \left(\mathrm{Q}_{\mathrm{obs}}(\mathrm{t})\right)-\log \left(\mathrm{Q}_{\mathrm{cal}}(\mathrm{t})\right)\right)^{2}}{\sum_{\mathrm{t}=1}^{\mathrm{nt}}\left(\log \left(\mathrm{Q}_{\mathrm{obs}}(\mathrm{t})\right)-\log \left(\overline{\mathrm{Q}_{\mathrm{obs}}}\right)\right)^{2}} \\
& \mathrm{E}(\%)=\frac{\sum_{\mathrm{t}=1}^{\mathrm{nt}}\left(\mathrm{Q}_{\mathrm{cal}}(\mathrm{t})-\mathrm{Q}_{\mathrm{obs}}(\mathrm{t})\right)}{\sum_{\mathrm{t}=1}^{\mathrm{nt}}\left(\mathrm{Q}_{\mathrm{obs}}(\mathrm{t})\right)} \times 100
\end{aligned}
$$

Em que: $\mathrm{t}=$ intervalo de tempo; $\mathrm{nt}=$ número de intervalos de tempo; $\mathrm{V}=$ volume $\left(\mathrm{m}^{3}\right) ; \mathrm{E}$ $(\%)=$ erro relativo adimensional deste volume em porcentagem; Qcal= vazão calculada (simulada) no posto; Qobs= vazão observada; e = média das vazões observadas.

Os coeficientes ENS e ENS ${ }_{\log }$ podem variar a partir de negativo infinito até 1, sendo 1 um ajuste perfeito. O valor de ENS é fortemente influenciado por erros nas vazões máximas e o de ENSlog, nas vazões mínimas. Para o erro percentual de volumes E (\%), valores negativos indicam que o modelo está subestimando a vazão observada; enquanto que, para valores positivos, o modelo está superestimando a vazão observada, e quanto mais próximos de zero, menor o erro geral do modelo. Oliveira (2014) utilizou uma classificação dos resultados das variáveis mencionadas, conforme Tabela 3.

Para avaliar a influência dos diferentes cenários na vazão da bacia hidrográfica do rio Pardo, foram criados dois projetos para a simulação, utilizando, para isso, os mesmos parâmetros calibrados e os períodos da calibração e validação (janeiro de 2000 a dezembro de 2010), com um projeto utilizando as CRHs de 2001, e o outro, as CRHs de 2016. 
Para avaliar se houve diferenças significativas entre os dados de vazão para os dois cenários, verificou-se inicialmente a normalidade dos dados pelo teste de Anderson-Darling, em que as hipóteses a serem testadas foram: H0, os dados seguem uma distribuição normal; e H1, os dados não seguem uma distribuição normal.Em caso de rejeição de H0, os dados foram transformados por meio da técnica Box Cox, com o objetivo de utilização da estatística paramétrica. Após essa etapa, avaliou-se a homogeneidade dos dados pelo teste $\mathrm{F}$ e então aplicou-seteste $\mathrm{t}$ de Student para amostras dependentes (teste t pareado). As hipóteses testadas foram: H0, a média das diferenças na população é igual a zero; e H1, a média das diferenças na população não é igual a zero. Após rejeição de H0, avaliaram-se as alterações nas vazõesanuais médias, mínimas e máximas.

Tabela 3 - Classificação dos índices de desempenho

\begin{tabular}{lcc}
\hline \multicolumn{1}{c}{ Classificação } & ENS e ENSIog & E(\%) \\
Muito bom & $0,75<$ ENS e ENSlog $\leq 1,00$ & $E(\%)< \pm 10$ \\
Bom & $0,65<$ ENS e ENSlog $\leq 0,75$ & $\pm 10 \leq \mathrm{E}(\%)< \pm 15$ \\
Satisfatório & $0,50<$ ENS e ENSlog $\leq 0,65$ & $\pm 15 \leq \mathrm{E}(\%)< \pm 25$ \\
Insatisfatório & ENS e ENSlog $\leq 0,50$ & $\mathrm{E}(\%) \geq \pm 25$ \\
\hline
\end{tabular}

Fonte: Oliveira (2014)

\section{RESULTADOS E DISCUSSÃO}

\subsection{Calibração e validação}

Os resultados da análise de desempenho para o período de calibração indicaram que as estações 53540001 e 53650000 apresentaram desempenho insatisfatório ao representar as vazões de cheia e estiagem, de acordo com os índices ENS e ENSlog, respectivamente; e os $\mathrm{E}(\%)$ de volume apresentaram superestimativas e subestimativas dentro de um limite muito bom (Tabela 4). A estação 53880000 representou de forma satisfatória as vazões de cheias e, com bom desempenho, as vazões de estiagem durante a calibração. Além disso, apresentou superestimativa dentro de um limite muito bom no erro total do volume. 
Tabela 4 - Índices de desempenho do MGB-IPH para três estações localizadas na bacia do rio Pardo, Brasil

\begin{tabular}{|c|c|c|c|c|c|}
\hline Estação & $\begin{array}{c}\text { Área monitorada } \\
\left(\mathrm{km}^{2}\right)\end{array}$ & Sub-bacia & Parâmetro & Calibração & Validação \\
\hline & & & ENS & 0,421 & 0,119 \\
\hline \multirow[t]{3}{*}{53540001} & 10791,721 & 1 & ENSlog & 0,296 & $-0,995$ \\
\hline & & & $E(\%)$ & 2,711 & 6,033 \\
\hline & & & ENS & 0,333 & 0,236 \\
\hline \multirow[t]{3}{*}{53650000} & 18439,686 & 2 & ENSlog & 0,36 & $-0,329$ \\
\hline & & & E (\%) & $-8,194$ & $-8,037$ \\
\hline & & & ENS & 0,635 & 0,530 \\
\hline \multirow[t]{2}{*}{65880000} & 29284,012 & 3 & ENSlog & 0,722 & 0,652 \\
\hline & & & $E(\%)$ & 2,687 & 4,313 \\
\hline
\end{tabular}

Fonte: Autores (2020)

É importante destacar que a calibração realizada para as sub-bacias a montante influencia a calibração das sub-bacias a jusante, ou seja, o resultado da calibração e validação observado na estação 53880000 inclui também as outras sub-bacias, pois esta localiza-se mais a jusante e monitora uma área de captação de $29.284,012$ km² $(89,70 \%)$ dos $32.649 \mathrm{~km}^{2}$ totais.

Ao realizar a calibração do modelo MGB-IPH na bacia Araguaia, com propagação Muskingum-Cunge, Pontes (2015) observou nas estações Conceição do Araguaia, Xambioá e Araguatins os valores de ENS de, respectivamente, 0,55, 0,63 e 0,70; ENSlog de $0,86,0,85$ e 0,88 , com os $E(\%)$ sendo 18,02, 28,98 e 24,71.Essas três estações, assim como neste estudo, estão monitorando sub-bacias no sentindo da jusante, e percebese o comportamento semelhante de aumento nos índices ENS e ENSlog à medida que as estações se aproximam da foz. Percebe-se também que os valores de ENSlog foram melhores que os obtidosneste estudo, mas por outro lado, os E (\%) foram inferiores. Ao utilizar o MGB-IPH na bacia do rio Uruguai, Guimarães (2018) encontrou a calibração NSE variando de 0,74 a 0,89, NSlog de 0,75 a 0,89 e E (\%) de -10,2 a 6,4.

Os valores de ENS e E (\%) observados neste estudo foram superiores aos encontrados por Pimentel (2017), que, ao utilizar o modelo conceitual semi- 
distribuído(SWAT) na modelagem hidrológica da bacia do rio Jucu, no Espírito Santo, encontrou valores de ENS de 0,40 e E (\%) de 28,82, para a escala diária; e ENS de 0,60 e E (\%) de 11,83, para a escala mensal na etapa de calibração.

Para a validação (Tabela 4), as estações 53540001 e 53650000 representaram, de forma insatisfatória, as vazões de cheia e estiagem. Esse comportamento era esperado ao observar os resultados da calibração, sendo que os erros totais de volume permaneceram dentro de um limite muito bom. A estação 53880000 foi validada com desempenho satisfatório ao representar tanto as vazões de cheia quantode estiagem, mantendo-se o erro total dos volumes dentro do limite muito bom, obtido na calibração. Ao avaliar o desempenho geral do modelo, pode-se perceber que houve uma queda de desempenho da calibração para a validação.

Os resultados obtidos para a estação 53880000 foram semelhantes aos de Jati e Silva (2017), que ao aplicar o MGB-IPH para a bacia do rio Curuá-Uma, no Pará, observaram para a etapa de validação,com um período de 6 anos, ENS de aproximadamente 0,53; ENSlog de 0,63; e E (\%) de -6,00\%. Também durante a etapa de validação do MGB-IPH, Guimarães (2018) obteve NSE entre 0,47 e 0,83; NSlog de 0,40 a 0,80; e $E(\%)$ de $-21,5$ a 24,1. Os autores observaram que os resultados da validação foram, no geral, inferiores aos obtidos na validação, comportamento semelhante ao observado neste estudo. Para a estação 53880000, os valores de ENS e E (\%), na validação, foram superiores aos encontrados por Pimentel (2017), que para a escala diária observou ENS de 0,52 e E (\%) de 22,11; e para a escala mensal, ENS de 0,50 e E (\%) de $14,83$.

Ao analisar o hidrograma da Figura 2A, percebe-se uma subestimativa dos valores de picos de vazões máximas comdiferenças de 663,82 m³/s (fevereiro de 2004) e 314,08 m³/s (dezembro de 2000), no período de calibração; e diferenças de 660,35 m³/s (março de 2010), 443,24 m³/s (março de 2006) e 342,09 m³/s (janeiro de 2009), nos picos de vazões máximas, no período de validação (Figura 2B). Comportamento semelhante foi observado nos hidrogramas de calibração e validação encontrados por Collischonn et al. (2011), para o MGB-IPH, e por Oliveira (2014) e Pimentel (2017), para o modelo SWAT. 
Figura 2 - Vazões observadas e simuladas e o hietograma de precipitação para os períodos de calibração (A) e validação (B) da estação fluviométrica 53880000, da bacia do rio Pardo, Brasil

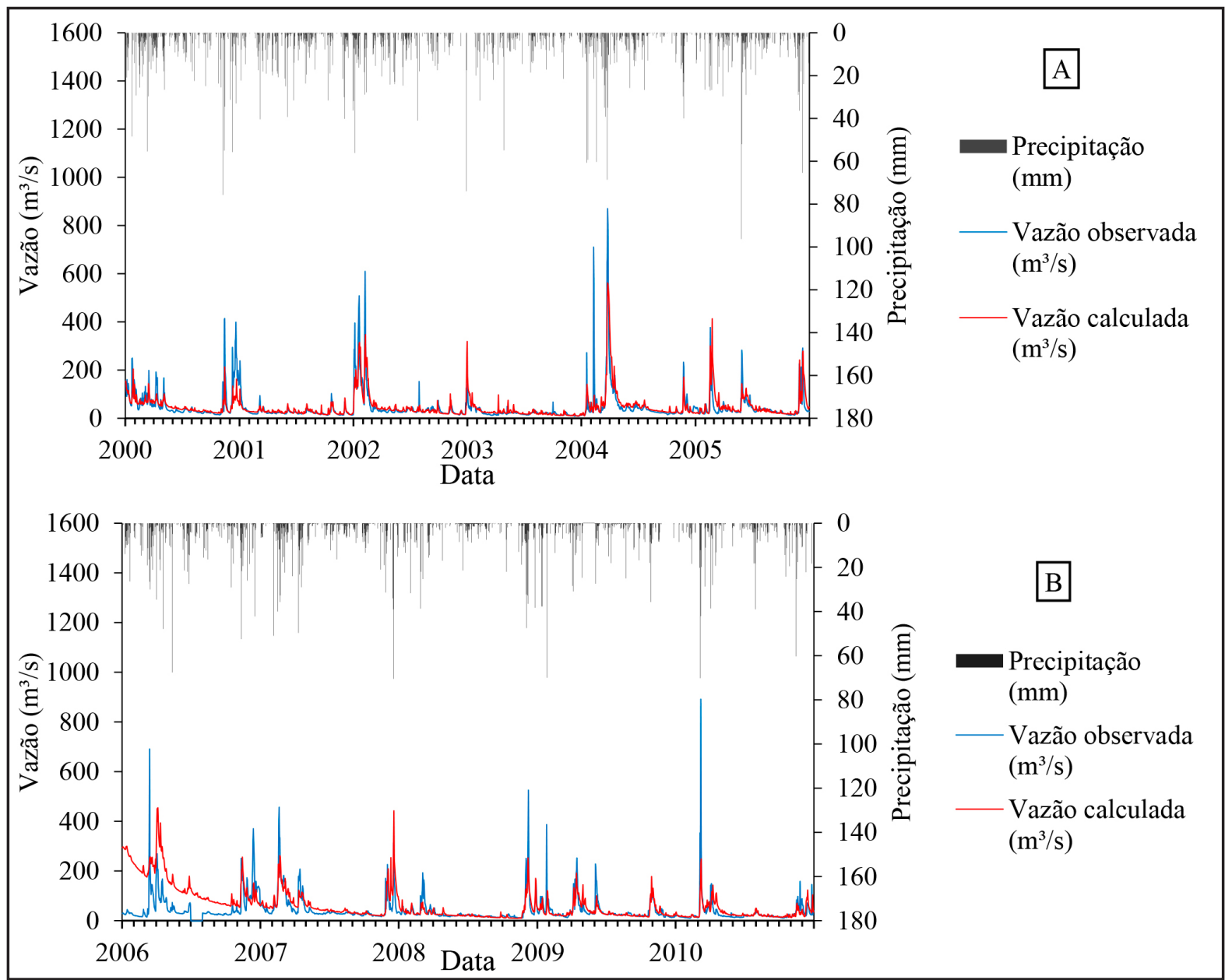

Fonte: Autores (2020)

Alguns argumentos para as grandes diferenças encontradas de subestimativas nos picos devazãopodem estar relacionados à presença de períodos com inconsistências nas vazões observadas. Harmel, Smith e Migliaccio (2010) relatam que a incerteza de mensuração nos dados de vazão, como a presença de falhas e os curtos registros de vazão, também podem resultar em problemas na modelagem hidrológica. Dessa forma, um melhor ajuste poderia ser obtido mediante a um número mais elevado de dados observados de vazão, e também de uma melhor consistência das séries de dados observados (OLIVEIRA, 2014). Em suma, mesmo com as diferenças observadas, o modelo MGB-IPH conseguiu reproduzir de forma satisfatória o comportamento geral 
do hidrograma observado, em termos da sazonalidade das vazões, da diferenciação de anos mais úmidos e anos mais secos; do instante de tempo de ascensão e recessão dos hidrogramas; e dos picos de cheias de média e pequena intensidade.

\subsection{Comparação entre os cenários validados}

As estatísticas mencionadas foram aplicadas somente à estação 53880000 , que foi validada de forma satisfatória. Os resultados do teste de Anderson-Darling concluíram, para um nível de $95 \%$ de confiança, que os dados diários das vazões simuladas para os cenários CRHs2001 e CRHs2016 não apresentaram distribuição normal. Desse modo, para a utilização das estatísticas paramétricas, os 4.015 valores de vazão diária foram alterados para 132 dados de vazões mensais médias, totais, máximas e mínimas, que, por fim, foram transformados por meio da técnica Box Cox para um $\lambda=-0,5$. Em seguida, aplicou-se o teste $\mathrm{F}$ para $95 \%$ de confiança, concluindo-se que as variâncias das vazões mensais médias, totais, máximas e mínimas transformadas são homogêneas. Aplicou-se então o teste t para amostras dependentes, de modo que se obtevediferença significativa entre as médias das vazões mensais médias, totais, máximas e mínimas para 95\% de confiança (Tabela 5).

Por meio do teste $t$, verificou-se que as médias das vazões mensais médias, totais, máximas e mínimas do cenário CRHs2016 foram superiores às do CRHs2001. Assim, os dados mensais foram transformados em vazões anuais médias, mínimas e máximas, por período de cheia e estiagem.

Tabela 5 - Resultados do teste t para amostras dependentes para os dados das vazões mensais médias, totais, máximas e mínimas dos diferentes cenários simulados pelo MGB-IPH, para a bacia do rio Pardo, Brasil

\begin{tabular}{lccccc}
\hline Cenários & $\mathbf{n}$ & Média $\left(\mathbf{m}^{\mathbf{3}} \mathbf{s}\right)$ & Total $\left(\mathbf{m}^{\mathbf{3}} \mathbf{s} \mathbf{s}\right)$ & Mínima $\left(\mathbf{m}^{\mathbf{3}} \mathbf{s}\right)$ & Máxima $\left(\mathbf{m}^{\mathbf{3}} \mathbf{s}\right)$ \\
\hline CRHs & 132 & $33,606 \mathrm{a}$ & $1022,040 \mathrm{a}$ & $22,041 \mathrm{a}$ & $59,446 \mathrm{a}$ \\
2001 & & & & & \\
CRHs & 132 & $34,889 \mathrm{~b}$ & $1061,710 \mathrm{~b}$ & $22,741 \mathrm{~b}$ & $62,098 \mathrm{~b}$ \\
2016 & & & &
\end{tabular}

Fonte: Autores (2020)

Em que: valores seguidos pela mesma letra na coluna não apresentam diferença significativa para $95 \%$ de confiança.

Ao analisar os picos de vazão para o período de cheias (Figura 3A) e estiagem 
(Figura 3B), percebe-se que o cenário CRHs2016 apresenta valores iguais ou superiores ao CRHs2001. Para o período de cheia, o cenário CHRs2016 apresentou um volume 2,92\% superior, com os anos de 2002, 2004 e 2005 não apresentando diferença entre os cenários; e os anos 2000 e 2007 apresentando, respectivamente, vazões de 26,51 e 30,45 m³/s maiores. Para o período de estiagem, o volume do cenário CRHs2016 foi 6,20\% superior, com o ano 2001 não apresentando diferença entre os cenários, e 2005 e 2006 apresentando valores de vazões 12,70 e 12,88 m³/s superiores no CRHs2016.

Os resultados para os picos de vazão podem ser explicados pela diminuição de áreas de floresta e aumento de áreas dedicadas a atividades agropecuárias e sem cobertura vegetal, no cenário das CRHs de 2016. Como explicam Lotz, Opp e He (2017), ao estudarem a influência de diferentes cenários de uso e ocupação do solo nos escoamentos da bacia Dongting Lake, na China, através do modelo SWAT, constatouse que o cenário com maior porcentagem de vegetação florestal demonstrou aumentos nas taxas de evapotranspiração e diminuição do escoamento superficial, o que acarretou uma menor disponibilidade de água, semelhante ao observado para os resultados do cenário CRHs2001 neste trabalho. Para a situação de diminuição de áreas de florestas, Aguiar et al. (2018) concluíram que, para cenários com redução na cobertura por vegetação nativa em áreas de preservação permanente, os picos de vazão simulados pelo modelo HEC-HMS tiveram acréscimo de até 30,7\%; resultados que corroboram os encontrados neste estudo, para o cenário CRHs2016.

Figura 3 - Pico de vazões anuais simuladas pelo modelo MGB-IPH para os períodos de cheia (A) e estiagem (B), para a estação fluviométrica 53880000, da bacia do rio Pardo, Brasil

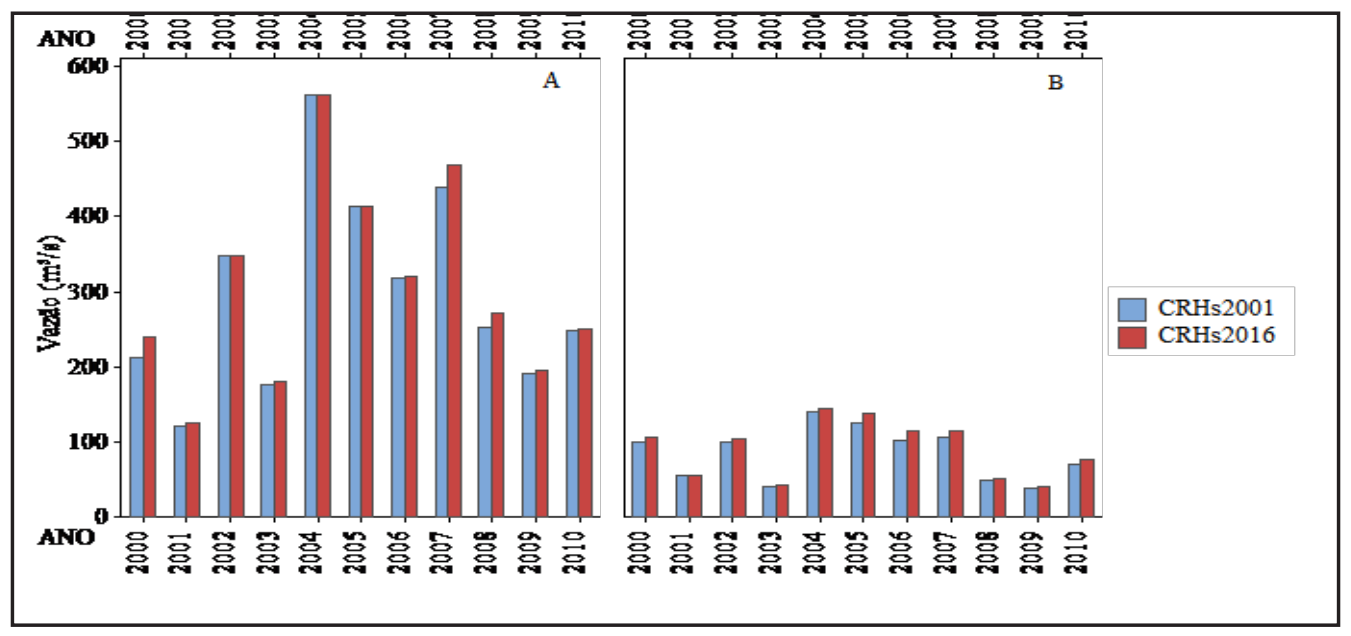

Fonte: Autores (2020) 
No que tange às modificações nas áreas agropecuárias, é importante destacar que a depender do sistema de cultivo utilizado, os impactos serão diferentes. No caso de sistemas convencionais de plantio, a alteração no escoamento é mais intensa em comparação com o plantio direto. Napoli, Massetti e Orlandini (2017), ao analisarem a dinâmica do escoamento superficial na bacia do rio Elsa, na província de Florence, na Itália, utilizando o modelo SWAT, considerando 50 anos de mudanças no uso e ocupação do solo, concluíram que o aumento de áreas de agricultura, onde eram praticados sistemas de cultivos não conservacionistas, como o plantio convencional,estava associado a aumentos nos picos de vazão, sendo estes advindos do aumento do escoamento superficial.

É sabido que o solo exposto no preparo para o plantio e os vários estágios de desenvolvimento das culturas anuais podem modificar a dinâmica do escoamento superficial ao longo do ano, o que acaba refletindo nos picos de vazão (ZHAO et al., 2014). Essa afirmação é exemplificada também nosestudos de Aguiar (2017), que avaliou a dinâmica sazonal da cobertura do solo agrícola na geração do escoamento superficial, por meio do modelo HEC-HMS, na bacia drenada pelo alto vale do rio Marrecas, no Paraná, concluindo que a relação entre uso do solo e escoamento superficial foi quantificada por taxas de aumento nos picos de vazão, que variaram de 0,78 a 1,64 $\mathrm{m}^{3} / \mathrm{s}$ por $\mathrm{km}^{2}$ de solo exposto.

Por meio desses estudos, é possível que os maiores picos de vazões observados no cenário CRHs2016 possam estar relacionados ao aumento de áreas de cultivo que utilizam sistemas não conservacionistas de preparo do solo ou pela intensificação do pastejo, entre 2001 e 2016, na bacia do rio Pardo.

Os resultados deste trabalho diferem de Jati e Silva (2017), que verificaram que o cenário com diminuição de áreas de vegetação e aumento de áreas de solo exposto e antropização demonstrou picos de vazões menores quando comparados ao cenário inverso, embora os autores não tenham deixado clara a explicação desse comportamento. 
As vazões mínimas (Figura 4A) e médias (Figura 4B) anuais demonstram comportamento semelhante às máximas, com o cenário CRHs2016 apresentando vazões superiores ao CRHs2001. As vazões mínimas anuais do cenário CRHs2016 apresentaram volume total 3,56\% superior, com amplitude das diferenças de 0,26 m³/s, em 2008, a 0,59 m³/s, em 2006. Para as vazões médias anuais, o cenário CRHs2016 apresentou volume total 3,56\% superior, com amplitude das diferenças de 0,88 $\mathrm{m}^{3} / \mathrm{s}$, em 2003, a 2,16 m³/s, em 2005.

Mesmo havendo diferença significativa entre as vazões mensais dos diferentes cenários, ao observar os valores em escala anual, percebe-se que as diferenças são pequenas, não ultrapassando o valor de 2,2 m³/s para as médias, e de 0,6 m³/s para as mínimas. Pequenas variações nas vazões médias e mínimas também foram observadas por Pimentel (2017), que, ao avaliar a resposta da vazão ao incremento de áreas florestais, obteve uma variação muito pequena, menor que $1 \%$ de diferença entre os cenários de menor ( $56 \%$ da bacia com floresta) e maior ( $23 \%$ da bacia florestada) da Qmed simulada.

Figura 4 - Vazões mínimas (A) e médias (B) anuais simuladas pelo modelo MGB-IPH para a estação fluviométrica 53880000, da bacia do rio Pardo, Brasil

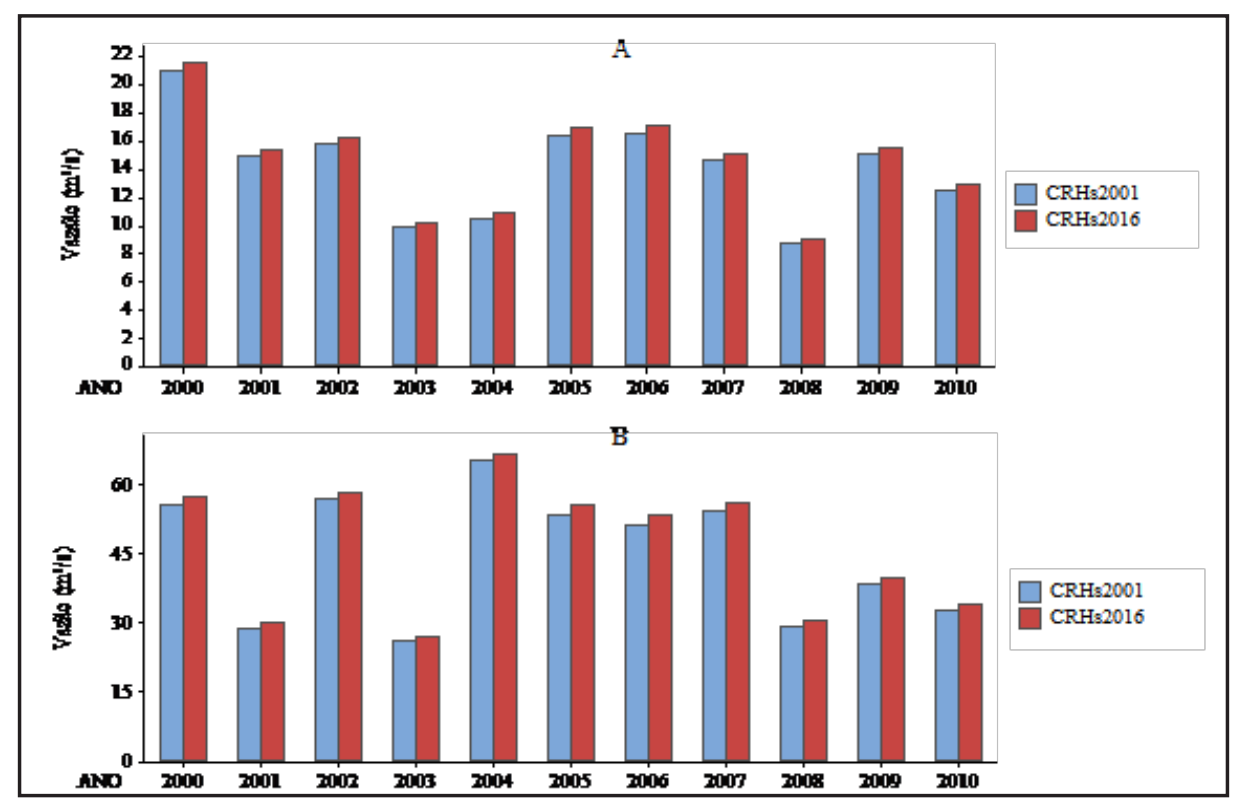

Fonte: Autores (2020) 
Os resultados obtidos neste trabalho, com o cenário com menor porcentagem de área coberta por florestas apresentando maiores valores de vazão, foram não esperados, pois é sabido que a cobertura com florestas é responsável por aumentar as vazões médias e/ou mínimas, pois ajuda na restauração e melhoria das funções hidrológicas do solo, auxiliando na infiltração, reduzindo o escoamento superficial, protegendo o solo da compactação, por meio da interceptação da água da chuva, e proporcionando a recarga dos aquíferos (MENDES, 2016; OLIVEIRA, 2014b). Porém, é importante destacar que o aumento de áreas de floresta também está associado ao aumento da evapotranspiração, bem como à retenção de água precipitada,ocasionada pela interceptação por meio do dossel. Nesse sentido, Pimentel (2017) também verificou diminuição da vazão para cenários com incremento de área de floresta, por meio do modelo SWAT, e destaca que, como a evapotranspiração e interceptação são expressivamente superiores às de outras classes, o impacto final sobre as vazões pode ser positivo, negativo ou inexpressivo.

Como exemplo de impacto negativo e diferente deste estudo, Rodrigues et al. (2015) observaram um aumento da vazão mínima para a simulação na bacia do vale do rio Pará, em Minas Gerais. Desse modo, conforme houve o acréscimo na área de floresta, foi apresentada uma maior vazão. Por outro lado, os resultados de Oliveira (2014b) e Smethurst et al. (2015) foram semelhantes aos deste trabalho, em que ocorre diminuição da vazão conforme há o aumento de área de floresta.

\section{CONCLUSÃO}

O MGB-IPH foi validado de forma satisfatória para a estação 53880000 da Agência Nacional de Águas, apresentando subestimativa de picos de vazões máximas, porém representou adequadamente os dados observados em termos da sazonalidade das vazões, da diferenciação de anos mais úmidos e anos mais secos e dos picos de cheias de média e pequena intensidade.

O modelo foi sensível ao identificar o impacto das mudanças do uso e ocupação do solo nas vazões simuladas para a bacia hidrográfica do rio Pardo, com o cenário de 2016 apresentando maiores valores de vazões mensais médias, totais, máximas e mínimas do que o cenário de 2001. 


\section{AGRADECIMENTOS}

Agradeço à Coordenação de Aperfeiçoamento de Pessoal de Nível Superior (CAPES) pela concessão de bolsa por meio do seu Programa de Demanda Social (DS) durante todo o período de realização deste trabalho.

\section{REFERÊNCIAS}

AGUIAR, W. de. Simulações hidrológicas de cenários de uso e ocupação do solo na bacia drenada pelo alto vale do rio Marrecas (PR). 2017. 108 f. Tese (Doutorado em Engenharia Agrícola) - Universidade Estadual do Oeste do Paraná. Cascavel, 2017.

AGUIAR, W. de; SAMPAIO, S. C.; PAISANI, J. C.; REIS, R. R. dos. Implicações nos picos de vazão perante as alterações no código florestal brasileiro. Engenharia Agrícola, [s.l], v. 38, n. 2, 2018.

BAYER, D. M.; COLLISCHONN, W. Análise de sensibilidade do Modelo MGB-IPH a mudanças de uso da terra. Revista Brasileira de Recursos Hídricos, [s.I], v. 18, p. 165-179, 2013.

BRAVO, J. M.; COLLISCHONN, W.; DA PAZ, A. R.; ALLASIA, D.; DOMECQ, F. Impact of projected climate change on hydrologic regime of the Upper Paraguay River basin. Climatic Change, [s.l], v. 127, p. 27-41, 2014.

BRITO NETO, R. L. Simulação hidrológica para a bacia hidrográfica do Rio Pardo em diferentes cenários de uso e ocupação do solo. 2018. 78 f. Dissertação (Mestrado em Ciências Florestais) - Universidade Estadual do Sudoeste da Bahia, Vitória da Conquista, 2018.

COLLISCHONN, B.; PAIVA, R. C. D. D.; COLLISCHONN, W.; MEIRELLES, F. S. C.; SCHETTINI, E. B. C.; FAN, F. M. Modelagem hidrológica de uma bacia com uso intensivo de água: Caso do Rio Quaraí-RS. Revista Brasileira de Recursos Hídricos, [s.I], v. 16, n. 4, p. 119-134, 2011.

COSTA, B. S. Modelagem hidrológica utilizando um sistema computacional: estudo de caso do Rio Cachoeira- Bahia. 2014. 121 f. Dissertação (Mestrado em Desenvolvimento Regional e Meio Ambiente) - Universidade Estadual De Santa Cruz, Ilhéus, 2014.

DE MELO, M. M. A. P. Modelagem da qualidade da água na bacia hidrográfica do Rio Piracicaba-MG. 2014. 164 f. Dissertação (Mestrado em Análise e Modelagem de Sistemas Ambientais) - Universidade Federal de Minas Gerais, Belo Horizonte, 2014.

DE MORISSON, V. M.; DE FÁTIMA R. D. Topodata: Brazilian full coverage refinement of SRTM data. Applied Geography, [s.I], v. 32, n. 2, p. 300-309, 2012.

FAN, F. M. Simulação dos impactos de lançamento de poluentes sobre a qualidade da água de bacias hidrográficas integrada com sistema de informação geográfica. 2013. 241 f. Dissertação (Mestrado em Recursos Hídricos e Saneamento Ambiental) - Universidade Federal do Rio Grande do Sul, Porto Alegre, 2013. 
FEITOSA, J. R.; FERNANDES, H. C.; TEIXEIRA, M. M.; CECON, P. R. Influência da pressão interna dos pneus e da velocidade de deslocamento nos parâmetros operacionais de um trator agrícola e nas propriedades físicas do solo. Engenharia Agrícola,Jaboticabal, v. 35, n. 1, p. 117-127, 2015.

GUIMARÃES, G. M. Análise da previsibilidade de cheias na bacia do rio Uruguai através do Modelo MGB-IPH. 2018.174 f. Monografia (Bacharelado em Engenharia Ambiental) - Instituto de Pesquisas Hidráulicas e Escola de Engenharia, Universidade Federal do Rio Grande do Sul, Porto Alegre, 2018.

HAGHNEGAHDAR, A.; TOLSON, B. A.; CRAIG, J. R.; PAYA, K. T. Assessing the performance of a semi-distributed hydrological model under various watershed discretization schemes. Hydrological Processes, [s.I.], v. 29, n. 18, p. 4018-4031, 2015.

HARMEL, R. D.; SMITH, P. K.; MIGLIACCIO, K. W. Modifying goodness-of-fit indicators to incorporate both measurement and model uncertainty in model calibration and validation. American Society of Agricultural and Biological Engineers, St. Joseph, v. 53, n. 1, p. 55-63, 2010.

JATI, D. A.; SILVA, J. T. da. Estudos geo-hidrológicos da bacia do rio Curuá-Una, Santarém, Pará: Aplicação do modelo hidrológico de grandes bacias (MGB-IPH). Revista Brasileira de Geografia Física, Recife, v. 10, n. 4, p. 1296-1311, 2017.

LOTZ, T.; OPP, C.; HE, X. Factors of runoff generation in the Dongting Lake basin based on a SWAT model and implications of recent land cover change. Quaternary International, [s.I.], v. 434, p. 1-9, 2017.

MAPBIOMAS. Projeto de Mapeamento Anual da Cobertura e Uso do Solo do Brasil, 2017. Algorithm Theoretical Base Document e Results - MapBiomas General "Handbook". Disponível em:<mapbiomas.org/pages/methodology>. Acesso em: 21 jun. 2018.

MELO, F. P. de. Risco ambiental e ordenamento do território em Garanhuns-PE. 2016. 246 f. Tese (Pós-Graduação em Geografia) - Universidade Federal de Sergipe, São Cristóvão, 2016.

MENDES, N. G. D. S. Estudo das vazões na bacia hidrográfica do rio Itapemirim. 2016. 98 f. Dissertação (Mestrado em Ciências Florestais) - Universidade Federal do Espírito Santo, Jerônimo Monteiro, 2016.

NAPOLI, M.; MASSETTI, L.; ORLANDINI, S. Hydrological response to land use and climate changes in a rural hilly basin in Italy. Catena, [s.I.], v. 157, p.1-11, 2017.

NÓBREGA, M. T.; COLLISCHONN, W.; TUCCI, C. E. M.; PAZ, A. R. Uncertainty in climate change impacts on water resources in the Rio Grande Basin, Brazil. Hydrology and Earth System Sciences, [s.I.], v. 15, p. 585-595, 2011.

OLIVEIRA, L. T. Aplicação do modelo SWAT para simular vazões em uma bacia hidrográfica em Aracruz, ES. 2014. 162 f. Dissertação (Mestrado em Ciências Florestais) - Universidade Federal do Espírito Santo, Espírito Santo, 2014. 
OLIVEIRA, K. D. Modelagem hidrológica da bacia do rio Jucu Utilizando o modelo DHSVM.2014. 177 f. Dissertação (Mestrado em Ciências Florestais) - Universidade Federal do Espírito Santo, Jerônimo Monteiro, 2014b.

PAIVA, R. C. D.; COLLISCHONN, W.; SCHETTINI, E. B. The Case Studies: Climate Change Impacts on Water Resources in the Quaraí river basin. In: FUNG, F.; LOPEZ, A.; NEW, M. Modelling the impact of climate change on water resources. New Jersey: Wiley-Blackwell, 2011.136-182 p.

PEREIRA, M.; KAYSER, R. B.; COLLISCHONN, W. Integração do Modelo Hidrológico para Grandes Bacias MGB-IPH e Sistemas de Informação Geográfica para suporte à decisão de outorga de direito de uso da água. Rega: revista de gestión del agua de America Latina, Porto Alegre, v. 9, n. 2 p. 21-33, 2012.

PIMENTEL, S. M. Modelagem hidrológica da bacia do rio Jucu sob diferentes cenários de cobertura florestal. 2017. 79 f. Dissertação (Mestrado em Ciências Florestais) - Universidade Federal do Espírito Santo, Espírito Santo, 2017.

PONTES, P. R.; COLLISCHONN, W.; FAN, F. M.; PAIVA, R. C.; BUARQUE, D. C. Modelagem hidrológica e hidráulica de grande escala com propagação inercial de vazões. Revista Brasileira de Recursos Hídricos, [s.I.],v. 20, n. 4, p. 888-904, 2015.

RODRIGUES, E. L.; ELMIRO, M. A. T.; JACOBI, C. M.; LAMOUNIER, W. L. Aplicação do modelo SWAT na avaliação do consumo de água em áreas de florestas plantadas na bacia do rio Pará, Alto São Francisco, em Minas Gerais. Sociedade \& Natureza, Uberlândia, v. 27, n. 3, p. 485500, 2015.

SANTOS, H. G.; CARVALHO JUNIOR, W. D.; DART, R. D. O.; ÁGLIO, M. L. D.; SOUSA, J. S. de.; PARES, J. G.; FONTANA, A.; MARTINS, A. L. S.; OLIVEIRA, A. P. de. O novo mapa de solos do Brasil: legenda atualizada. 2.ed. Rio de Janeiro: Embrapa Solos, 2011. 67 p.

SANTOS, L. C. O. Influência dos usos consuntivos da água e do uso e cobertura da terra na vazão da bacia hidrográfica do rio Pardo. 2017. 80 f. Dissertação (Mestrado em Ciências Florestais) - Universidade Estadual do Sudoeste da Bahia, Vitória da Conquista, 2017.

SILVA, V. P. R.; SILVA, M. T.; SOUZA, E. P. Influence of land use change on sediment yield: a case study of the sub-middle of the São Francisco river basin. Engenharia Agrícola, Jaboticabal, v. 36, n. 6, p. 1005-1015, 2016.

SILVA, W. M.; BIANCHINI, A.; CUNHA, C. A. Modeling and correction of soil penetration resistance for variations in soil moisture and soil bulk density. Engenharia Agrícola, Jaboticabal, v. 36, n. 3, p. 449-459, 2016.

SMETHURST, P. J.; ALMEIDA, A. C.; LOOS, R. A. Stream flow unaffected by Eucalyptus plantation harvesting implicates water use by the native forest streamside reserve. Journal of Hydrology: Regional Studies, [s.I.], v. 3, p. 187-198, 2015.

ZHAO, X.; HUANG, J.; WU, P.; GAO, X. The dynamic effects of pastures and crop on runoff and sediments reduction at loess slopes under simulated rainfall conditions. Catena, [s.l.], v. 119, p. 1-7, 2014. 


\section{Contribuição de Autoria}

\section{1 - Rodrigo Lacerda Brito Neto}

Engenheiro Florestal, Me.

https://orcid.org/0000-0001-7233-2192•britonetorl@gmail.com

Contribuição: Conceituação, Análise Formal, Validação, Investigação, Metodologia, Visualização, Escrita - primeira redação, Escrita - revisão e edição

\section{2 - Cristiano Tagliaferre}

Engenheiro Agrônomo, Dr., Professor

https://orcid.org/0000-0003-4776-473X•tagliaferre@uesb.edu.br

Contribuição: Conceituação, Curadoria de dados, Análise Formal, Administração do projeto, Supervisão, Validação, Visualização, Escrita - primeira redação, Escrita revisão e edição

\section{3 - Odair Lacerda Lemos}

Engenheiro Agrônomo, Dr., Professor

https://orcid.org/0000-0001-8347-8947•olemos@uesb.edu.br

Contribuição: Análise Formal, Supervisão, Validação, Visualização, Escrita - revisão e edição

\section{4 - Felizardo Adenilson Rocha}

Engenheiro Agrícola, Dr., Professor

https://orcid.org/0000-0001-6410-5176•felizardo@ifba.edu.br

Contribuição: Análise Formal, Supervisão, Validação, Visualização, Escrita - revisão e edição

\section{5 - Alessandro de Paula}

Engenheiro Florestal, Dr., Professor

https://orcid.org/0000-0003-3676-3846•apaula@uesb.edu.br

Contribuição: Análise Formal, Supervisão, Validação, Visualização, Escrita - revisão e edição

\section{Como citar este artigo}

Brito Neto, R. L.; Tagliaferre, C.; Lemos, O. L.; Rocha, F. A.; Paula, A. Aplicação do modelo MGBIPH na bacia hidrográfica do Rio Pardo em diferentes cenários de uso e ocupação do solo. Ciência Florestal, Santa Maria, v. 31, n. 1, p. 191-213, 2021. DOI 10.5902/1980509836095. Disponível em: https://doi.org/10.5902/1980509836095. Acesso em: xx mês abreviado 202x. 